\title{
SOBREVIVÊNCIA E CRESCIMENTO DE SETE ESPÉCIES ARBÓREAS NATIVAS EM UMA ÁREA DEGRADADA DE FLORESTA ESTACIONAL DECIDUAL, NORTE DE MINAS GERAIS ${ }^{1}$
}

Yule Roberta Ferreira Nunes², Nathalle Cristine Alencar Fagundes³, Maria das Dores Magalhães Veloso², Anne Priscila Dias Gonzaga ${ }^{4}$, Ellen Bárbara Santos Domingues ${ }^{5}$, Hisaías de Souza Almeida ${ }^{6}$, Gislene Carvalho de Castro $^{7}$ e Rubens Manoel Santos ${ }^{8}$

\begin{abstract}
RESUMO - Este trabalho objetivou avaliar o crescimento e sobrevivência de sete espécies arbóreas nativas em diferentes tratamentos, em uma área degradada na Reserva da COPASA (Juramento, MG). Para tal, foram utilizados aproximadamente 1,2 ha da área, onde foram plantadas 899 mudas, de sete espécies nativas (Anadenanthera colubrina, Copaifera langsdorffii, Dilodendron bipinatum, Myracrodruon urundeuva, Pterogyne nitens, Schinopsis brasiliensis e Senegalia polyphylla), acompanhadas durante 24 meses. A área foi dividida em cinco parcelas de 0,24 ha, que representaram os seguintes tratamentos: (T1) parcela semeada com capim (Brachiaria sp.); (T2) parcela onde as covas foram tratadas com condicionador de solo; (T3) parcela semeada com capim consorciado com leguminosa (Cajanus cajan); (T4) parcela semeada com leguminosa; e (T5) controle. A porcentagem de mortalidade foi maior no T2 e menor no T3, sendo as espécies C. langsdorffii $(43,66 \%)$ e $S$. brasiliensis $(11,64 \%)$ aquelas com maior e menor porcentagem de mortalidade, respectivamente. O crescimento (altura e diâmetro) das mudas foi maior no T2 $(24,32 \pm 26,05 \mathrm{~cm}$ e $0,51 \pm 0,37 \mathrm{~mm}$, respectivamente) e menor no $\mathrm{T} 1(10,82 \pm 22,57 \mathrm{~cm}$ e $0,26 \pm 0,27 \mathrm{~mm}$; respectivamente). As espécies com maior crescimento em altura foram A. colubrina e $S$. polyphylla, já $D$. bipinatum e $S$. brasiliensis apresentaram maior crescimento em diâmetro. Pterogyne nitens apresentou o menor crescimento, além de alta mortalidade, mostrando baixa capacidade de estabelecimento em ambientes degradados.
\end{abstract}

Palavras-chave: Restauração ambiental; Plantio de mudas: Mata seca.

\section{SURVIVAL AND GROWTH OF SEVEN NATIVE TREE SPECIES IN A DEGRADED AREA IN TROPICAL DRY FOREST, NORTHERN MINAS GERAIS}

\begin{abstract}
This study aimed to evaluate the growth and survival of seven native tree species in different treatments in a degraded area at COPASA Reserve (Juramento, MG). Approximately 1.2 ha area was used, where 899 seedlings were planted of seven native species (Anadenanthera colubrina, Copaifera langsdorffii, Dilodendron bipinatum, Miracrodruon urundeuva, Pterogyne nitens, Schinopsis brasiliensis and Senegalia polyphylla) followed for 24 months. The area was divided into five plots of 0.24 ha, which represented the following treatments: (T1) plot sown with grass (Brachiaria sp.); (T2) plot where the pits were treated
\end{abstract}

\footnotetext{
${ }^{1}$ Recebido em 17.10.2014 aceito para publicação em 07.07.2015.

${ }^{2}$ Universidade Estadual de Montes Claros, Centro de Ciências Biológicas e da Saúde, Departamento de Biologia Geral, Montes Claros, MG - Brasil.E-mail:<yule.nunes@unimontes.br>e < doraveloso13@gmail.com>.

${ }^{3}$ Universidade Estadual de Montes Claros, Centro de Estudos de Convivência com o Semiárido, Montes Claros, MG - Brasil. E-mail:<nath_fag@yahoo.com.br>.

${ }^{4}$ Universidade Federal dos Vales do Jequitinhonha e Mucuri, Faculdade Interdisciplinar em Humanidades, Campus JK, Diamantina, MG-Brasil. E-mail:<diaspri@gmail.com>.

${ }^{5}$ Instituto Federal de Educação Ciência e Tecnologia do Norte de Minas Gerais, IFNMG-Brasil. E-mail:<ellen.domingues@gmail.com>.

${ }^{6}$ Universidade Federal de Itajubá, Instituto de Recursos Naturais, Pinheirinho - Itajubá, MG - Brasil. E-mail: <hisaias37@gmail.com>.

${ }^{7}$ Universidade Federal de São João Del-Rei, Departamento de Ciências Naturais, São João del Rei, MG - Brasil. E-mail: <gislene.castro@gmail.com>.

${ }^{8}$ Universidade Federal de Lavras, Departamento de Ciências Florestais, Lavras, MG-Brasil. E-mail: <santosfloracaatinga@yahoo.com.br>.
} 
with soil conditioner; (T3) plot sown with grass and legumes (Cajanus cajan) together; (T4) plot sown with legumes; and (T5) control. The mortality percentage was higher in T2 and lower in T3, and the species C. langsdorffii (43.66\%) and S. brasiliensis (11.64\%) were the ones with higher and lower mortality rate, respectively. The growth (height and diameter) of seedlings was higher in T2 $(24.32 \pm 26.05 \mathrm{~cm}$ and 0.51 $\pm 0.37 \mathrm{~mm}$; respectively) and lowest in $T 1(10,82 \pm 22,57 \mathrm{~cm}$ and $0,26 \pm 0,27 \mathrm{~mm}$; respectively). The species with the largest growth in height were $\boldsymbol{A}$. colubrina and $\boldsymbol{S}$. polyphylla, and D. bipinatum and $\boldsymbol{S}$. brasiliensis showed the highest growth in diameter. Pterogyne nitens showed the lowest growth, and highest mortality, presenting low ability to establish in degraded environments.

Keywords: Environmental restoration; Seedlings planting; Dry forest.

\section{INTRODUÇÃO}

As vegetações tropicais vêm sendo destruídas pela ação humana a uma velocidade preocupante, principalmente pelos processos inevitáveis do crescimento populacional e econômico (GARCÍA-ORTH; MARTÍNEZ-RAMOS, 2011). Assim, atualmente a recuperação de áreas degradadas tem sido considerada prioritária, em face dos sérios danos ambientais decorrentes de ações antrópicas sobre os ecossistemas naturais (PALMER et al., 2007; DAVIES, 2010; RONI; BEECHIE, 2013). A retirada da cobertura vegetal, além de expor o solo, favorecendo a perda de sua fertilidade, sua desagregação e desestruturação (cujas partículas carreadas afetam a qualidade da água), causa danos à fauna associada e à biodiversidade total e descaracteriza a paisagem (BARON et al., 2002).

Em ecossistemas naturais degradados, a intervenção técnica adequada permite que os mecanismos de regeneração natural se tornem efetivos (ANDEL; ARONSON, 2012). Martins (2009) afirmou que o primeiro interesse da recuperação de áreas impactadas é promover o rápido crescimento de uma cobertura vegetal, de modo a controlar os processos erosivos, de assoreamento de mananciais de água e melhorar a fertilidade do solo. Nesse contexto, os projetos de recomposição de áreas degradadas objetivam, principalmente, o retorno da forma e função do ecossistema (ANDEL; ARONSON, 2012; CLEWELL; ARONSON, 2013). De fato, o uso de espécies nativas é reconhecido em projetos de recuperação, principalmente devido ao alto valor adaptativo dessas espécies às condições locais, permitindo, em curto prazo, proteção e enriquecimento do solo, abrigo e alimento para a fauna, perenização do regime hídrico e recuperação da paisagem (SANSEVERO et al., 2011). Desse modo, nos processos de revegetação, a recuperação da forma original do ecossistema e, ou, de uma ou mais funções desse sistema pode ser atingida e deve ser priorizada.
Apesar do aumento do interesse na preservação dos recursos naturais e do conhecimento ecológico das espécies nativas, as atividades de recuperação de áreas degradadas no Brasil são bastante recentes (MARTINS, 2009). Além do desmatamento e da perda das espécies nativas, estima-se que mais de 200 milhões de hectares de solo foram degradados no Brasil, principalmente por atividades como mineração, construção de estradas, utilização de métodos agropecuários impróprios e pela construção de barragens e hidroelétricas (ALVES et al., 2012).

As obras de engenharia, como a construção de barragens e hidrelétricas, em que ocorre remoção total da camada vegetal e de expressivas quantidades de material pedológico, dão origem às chamadas áreas de empréstimo (ALVES; SOUZA, 2008). Tais áreas são caracterizadas pela remoção total da vegetação original e pela retirada da camada superficial do solo, expondo o horizonte C e causando compactação, perda de matéria orgânica e baixa disponibilidade de nutrientes (ALVES et al., 2012). As áreas de empréstimo constituem, portanto, um ambiente altamente degradado, uma vez que tiveram a eliminação da vegetação e dos meios bióticos de regeneração natural (chuva de sementes, banco de sementes e de plântulas), comprometendo fortemente a resiliência do ambiente onde foram implantadas (MÜLLER, 1995; CHADA et al., 2004). Assim, a reabilitação desses ambientes é bastante lenta e, muitas vezes, imperceptível, equivalendo a várias gerações humanas (RODRIGUES et al., 2007). Nesse caso, o uso de medidas biológicas que acelerem os processos de construção dos ecossistemas deve ser priorizado (RODRIGUES et al., 2007).

A construção da barragem do rio Juramento, em Juramento, Norte de Minas Gerais, enquadra-se nesse tipo de processo de degradação, cujo solo da área de empréstimo, de cerca de 15 ha, foi utilizado na construção da barragem da represa. Após 23 anos da implantação 
da barragem (construída em 1981), a Área de Empréstimo apresentou retardo no processo de regeneração natural, com grandes áreas de solo descoberto e indícios de colonização inicial por espécies pioneiras, como algumas gramíneas e outras espécies herbáceas e por poucas espécies lenhosas, principalmente por Solanum lycocarpum St. Hil. (fruta-do-lobo). Utilizando sete espécies arbóreas nativas de alto valor fitossociológico na área (MELO et al., 2000), este trabalho teve como objetivo avaliar o crescimento e sobrevivência das mudas dessas espécies em diferentes tratamentos, em função do consórcio estabelecido com outras espécies vegetais e com um condicionador de solo, durante dois anos, nessa área degradada.

\section{MATERIAL E MÉTODOS}

\subsection{Caracterização da área de estudo}

Este estudo foi realizado na Área de Preservação da Companhia de Saneamento de Minas Gerais COPASAS.A. (1646’20”'S e 4339' 56”W), no Município de Juramento, MG. Essa área de preservação é formada por um reservatório que corresponde a $7,63 \mathrm{~km}^{2}$ de área inundada e, no entorno da represa, uma área de vegetação nativa equivalente a $23,37 \mathrm{~km}^{2}$ (NUNES et al., 2005). Aregião está incluída na transição dos domínios do Cerrado e da Caatinga e apresenta a Floresta Estacional Decidual e o Cerrado Sentido Restrito como principais fitofisionomias (NUNES et al., 2005). O solo na região é classificado como Argissolo Vermelho-Amarelo Eutrófico Típico, com horizonte A moderado, textura argilosa e horizonte B textural; e como Latossolo Vermelho-Amarelo Distrófico Típico, com horizonte A moderado, textura argilosa, com horizonte B latossólico apresentando espessura mínima de $50 \mathrm{~cm}$ (PVAe9) (EMBRAPA, 2013). O clima é do tipo subúmido seco, com estações seca e chuvosa bem definidas (NUNES et al., 2012). A temperatura média anual encontra-se em torno dos $23^{\circ} \mathrm{C}$, e a precipitação média é de 1.000 $\mathrm{mm} / \mathrm{ano}$, com chuvas concentradas nos meses de novembro a janeiro (NUNES et al., 2005).

Olocal denominado Área de Empréstimo foi selecionado para implantação do experimento, devido aos indícios que demonstraram retardo no processo de regeneração natural e à grande extensão desse local (15 ha). Nessa área, retiraram-se o horizonte orgânico do solo e grande parte do horizonte mineral superficial, sendo as camadas de solo utilizadas na construção da barragem da represa.

\subsection{Espécies selecionadas e modelo de recomposição}

Foram selecionadas sete espécies importantes da flora local: angico (Anadenanthera colubrina (Vell.) Brenan - Fabaceae), aroeira (Myracroduon urundeuva Allem. - Anacardiaceae), carne-de-vaca (Pterogyne nitens Tul. - Fabaceae), mamoninha (Dilodendron bipinnatum Radlk. - Sapindaceae), pau-d'óleo (Copaifera langsdorffii Desf. - Fabaceae), pau-preto (Schinopsis brasiliensis Engl - Anacardiaceae) e periquiteira (Senegalia polyphylla (DC.) Britton \& Rose-Fabaceae), através de um estudo fitossociológico em floresta adjacente (MELO et al., 2000). Para produção de mudas, foi feita a coleta de sementes das espécies na área de estudo de pelo menos 20 árvores reprodutivas, por espécie. As sementes coletadas foram beneficiadas e colocadas para germinar em sacos plásticos de polietileno de $3 \mathrm{~kg}$, em um viveiro de mudas localizado na área de estudo. As mudas foram regadas diariamente até próximo do período de plantio e, em sequência, aclimatadas.

Em dezembro de 2001, foi demarcado 1,2 ha na Área de Empréstimo, onde foi realizado o gradeamento do solo (trator e grade de $20 \mathrm{~cm}$ ), bem como feita a abertura das covas mecanicamente (trator e furador de $50 \mathrm{~cm}$ ), sendo demarcadas na área, posteriormente, cinco parcelas de 0,24 ha. Nessas parcelas, foram plantadas, no total, 899 mudas, sendo 190 de P. nitens, 155 de $M$. urundeuva, 145 de $S$. brasiliensis, 130 de D. bipinnatum, 122 de $S$. polyphylla, 89 de $A$. colubrina e 68 de $C$. langsdorffii. O número de mudas de cada espécie variou conforme a disponibilidade dessas na época de plantio, sendo distribuídas de forma igualitária e arbitrária. As mudas, marcadas e numeradas, foram plantadas em espaçamento de $3 \mathrm{~m} \times 3 \mathrm{~m}$ e acompanhadas, de fevereiro de 2002 a janeiro de 2004 (mensalmente até novembro de 2002 e trimestralmente a partir dessa data), por meio das medidas de altura total, com metro graduado, e do diâmetro à altura do solo (DAS), com paquímetro.

As parcelas estabelecidas corresponderam a cinco tratamentos distintos: (T1) plantio de mudas para áreas semeadas com capim (Brachiaria sp.); (T2) plantio de mudas para covas tratadas com $100 \mathrm{~g}$ de condicionador de solo TerraCotten (polímero hidroabsorvente condicionador de solo); (T3) plantio de mudas para áreas semeadas com capim (Brachiaria $\mathrm{sp}$.), consorciado com leguminosa (Cajanus cajan (L) Millsp; feijão-guandu

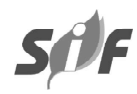

Revista Árvore, Viçosa-MG, v.39, n.5, p.801-810, 2015 
ou andu); (T4) plantio de mudas para áreas semeadas com leguminosa; e (T5) plantio de mudas para área sem tratamento de solo (controle). Tanto o capim quanto a leguminosa foram semeados manualmente, nas respectivas parcelas, cobrindo homogeneamente o solo e utilizando $1 \mathrm{~kg}$ de capim e $2,5 \mathrm{~kg}$ de leguminosa por parcela.

\subsection{Análise de Dados}

Para verificar a mortalidade das mudas plantadas, foi feita a porcentagem de mortalidade, pela razão entre o número de indivíduos mortos e o número total de indivíduos plantados, para cada espécie e tratamento. O crescimento dos indivíduos, tanto em altura quanto em diâmetro, foi obtido por meio da diferença entre a medida final e a medida inicial das mudas. Para detectar variações do crescimento entre as espécies, os tratamentos e a interação espécie $\times$ tratamento, foi utilizada a Análise de Variância (ANOVA), em procedimento GLM (Modelos Lineares Generalizados), seguida do pósteste de Tukey, no pacote estatístico R ( R DEVELOPMENT CORE TEAM, 2013), considerando cada indivíduo como repetição.

\section{RESULTADOS}

\subsection{Mortalidade das mudas}

A porcentagem de mortalidade das mudas variou entre as espécies, de 11,64 a 43,66\% (Tabela 1). As maiores porcentagens de mortalidade foram observadas em $C$. langsdorffii e $P$. nitens, sendo $S$. brasiliensis e $A$. colubrina as espécies com menores porcentagens de mortalidade. Além disso, o número de indivíduos mortos nos quatro primeiros meses após o plantio foi expressivo (15,35\%), chegando a $18,77 \%$ aos seis meses do plantio. No segundo ano, o incremento na mortalidade foi de $6,67 \%$, totalizando $25,44 \%$ de indivíduos mortos após 24 meses. Esses resultados indicaram que os primeiros meses de plantio (aqui expressos pelos quatro meses iniciais) representaram um período crítico à sobrevivência das mudas, após o que estas apresentam alta porcentagem de sobrevivência.

A porcentagem de indivíduos mortos também variou entre os tratamentos utilizados (Tabela 2). A menor mortalidade foi encontrada no tratamento consorciado entre capim e leguminosa (T3), enquanto a maior mortalidade foi encontrada no tratamento em que foi utilizado o condicionador de solo (T2). Pode-se observar que a mortalidade das espécies, dentro dos tratamentos, não apresentou padrão consistente. Para S. brasiliensis e $S$. polyphylla, a sobrevivência foi maior na área tratada com condicionador de solo (T2); para a $D$. bipinnatum, no tratamento com capim (T1); e no tratamento com leguminosa (T4); e para as demais espécies, no tratamento consorciado de capim e leguminosa (T3). Foi ainda observado que a mortalidade das plantas incorporadas foi acarretada, principalmente, por pisoteio de equinos, adicionado ao soterramento ocasionado por pequenas erosões formadas no período chuvoso.

\subsection{Crescimento das mudas}

O crescimento em diâmetro e em altura das espécies variou entre os diferentes tratamentos, entre as espécies e na interação tratamento $\times$ espécie (Tabela 3 ). Entre os tratamentos testados, as mudas plantadas no T2 (condicionador de solo) apresentaram maior crescimento médio em altura $(24,32 \pm 26,05 \mathrm{~cm})$ (Figura 1). No entanto, as mudas no $\mathrm{T} 1$ (capim) apresentaram menor crescimento em altura $(10,82 \pm 22,57 \mathrm{~cm})$. Do mesmo modo como foi observado no crescimento em altura, o crescimento em diâmetro $(0,51 \pm 0,37 \mathrm{~mm})$ das mudas plantadas no tratamento com T2 mostrou-se melhor e aquelas no $\mathrm{T} 1$, menor $(0,26 \pm 0,27 \mathrm{~mm})$.

Entre as espécies utilizadas no experimento, as espécies que apresentaram maiores crescimentos em diâmetro foram $D$. bipinnatum e $S$. brasiliensis, com médias de $0,56 \mathrm{~mm}( \pm 0,43)$ e $0,53 \mathrm{~mm}( \pm 0,35)$, respectivamente (Figura 2). Entretanto, A. colubrina e $S$. polyphylla apresentaram as maiores médias de crescimento em altura $(41,15 \pm 25,77 \mathrm{~cm}$ e $38,07 \pm$ $33,03 \mathrm{~cm}$, respectivamente). O contrário foi observado em $P$. nitens, que apresentou baixo crescimento em altura $(0,71 \pm 7,58 \mathrm{~cm})$ e diâmetro $(\mathrm{x}=0,12 \pm 0,12 \mathrm{~mm})$. Do mesmo modo, M. urundeuva cresceu pouco em altura $(8,27 \pm 14,32 \mathrm{~cm})$ e $C$. langsdorffii, em diâmetro $(0,27 \pm 14,32 \mathrm{~mm})$. Esses resultados indicaram variação interespecífica nas taxas de crescimento e a capacidade adaptativa das espécies ao ambiente alterado.

\section{DISCUSSÃO}

A sobrevivência e capacidade das mudas de se adaptarem ao ambiente dependem de complexa interação entre seus atributos morfológicos e fisiológicos, que determinam sua plasticidade fenotípica (GARWOOD, 1996). Dessa forma, a mortalidade depende da própria 
Tabela 1 - Número de indivíduos plantados, sobreviventes e mortos e porcentagem de mortalidade das mudas das sete espécies arbóreas utilizadas no reflorestamento na Reserva da COPASA (Juramento, MG), após dois anos (dezembro/2001 a janeiro/2004) de plantio.

Table 1 - Number of planted, surviving and dead individuals and mortality rate of seedlings of seven tree species used in reforestation in the COPASA Reserve (Juramento, MG) after two years of planting (December/2001 to January/ 2004).

\begin{tabular}{|c|c|c|c|c|}
\hline Espécie & Total plantado & Plantas sobreviventes & Plantas mortas & Mortalidade(\%) \\
\hline Anadenanthera colubrina & 89 & 76 & 13 & 14,77 \\
\hline Copaifera langsdorffii & 71 & 40 & 31 & 43,66 \\
\hline Dilodendron bipinnatum & 129 & 98 & 31 & 26,36 \\
\hline Myracroduon urundeuva & 162 & 124 & 38 & 23,46 \\
\hline Pterogyne nitens & 194 & 126 & 68 & 35,05 \\
\hline Schinopsis brasiliensis & 146 & 129 & 17 & 11,64 \\
\hline Senegalia polyphylla & 125 & 90 & 35 & 28,00 \\
\hline Total & 916 & 683 & 233 & 25,44 \\
\hline
\end{tabular}

Tabela 2 - Porcentagem de indivíduos mortos das mudas das sete espécies arbóreas nos diferentes tratamentos de cobertura de solo, na Reserva da COPASA (Juramento, MG), após dois anos de plantio (dezembro/2001 a janeiro/2004). T1 = áreas semeadas com capim; T2 = covas tratadas com condicionador de solo; T3 = áreas semeadas com capim consorciado com leguminosa; T4 = áreas semeadas com leguminosa; e T5 $=$ controle.

Table 2 - Percentage of dead individuals of seven tree species in the different treatments of soil cover in the COPASA Reserve (Juramento, MG) after two years of planting (December/2001 to January/2004). T1 = areas sown with grass; $T 2=$ pits treated with soil conditioner; T3 = areas sown with grass and legumes together; T4 = areas sown with legumes; and T5 = control.

\begin{tabular}{lccccc}
\hline \multirow{2}{*}{ Espécie } & \multicolumn{5}{c}{ Tratamentos $(\%$ mortalidade $)$} \\
\cline { 2 - 6 } & $\mathrm{T} 1$ & $\mathrm{~T} 2$ & $\mathrm{~T} 3$ & $\mathrm{~T} 4$ & $\mathrm{~T} 5$ \\
\hline Anadenanthera colubrina & 3,37 & 6,74 & 0 & 1,12 & 3,37 \\
Copaifera langsdorffii & 8,45 & 5,63 & 15,49 & 7,04 & 7,04 \\
Dilodendron bipinnatum & 3,88 & 6,20 & 5,43 & 3,88 & 6,98 \\
Myracroduon urundeuva & 3,09 & 8,03 & 1,23 & 5,56 & 5,56 \\
Pterogyne nitens & 7,22 & 7,22 & 3,61 & 9,28 & 7,73 \\
Schinopsis brasiliensis & 3,43 & 3,43 & 0 & 3,43 & 1,37 \\
Senegalia polyphylla & 5,60 & 3,20 & 4,00 & 7,20 & 8,00 \\
Total & 35,03 & 40,44 & 29,76 & 37,50 & 40,05 \\
\hline
\end{tabular}

Tabela 3 - Análise de Variância (ANOVA), em procedimento GLM (Modelos Lineares Generalizados), do crescimento das mudas das sete espécies nos diferentes tratamentos, após dois anos de plantio (dezembro/2001 a janeiro/2004), na Reserva da COPASA (Juramento, MG).

Table 3 - Analysis of Variance (ANOVA) in GLM (Generalized Linear Models) of seedlings growth of seven tree species in the different treatments after two years of planting (December/2001 to January/2004) in the COPASA Reserve (Juramento, $M G$ ).

\begin{tabular}{lccccc}
\hline \multirow{2}{*}{ Variáveis } & \multicolumn{3}{c}{ Diâmetro } & \multicolumn{2}{c}{ Altura } \\
\cline { 2 - 5 } & $\mathrm{gl}$ & $\mathrm{F}$ & $\mathrm{p}$ & $\mathrm{F}$ \\
\hline Tratamentos & 4 & 7,034 & $<0,001$ & 4,382 & $<0,01$ \\
Espécies & 6 & 29,441 & $<0,001$ & 57,691 & 2,09 \\
Tratamento $\times$ espécie & 24 & 2,128 & $=0,001$ & $<0,001$ \\
\hline
\end{tabular}




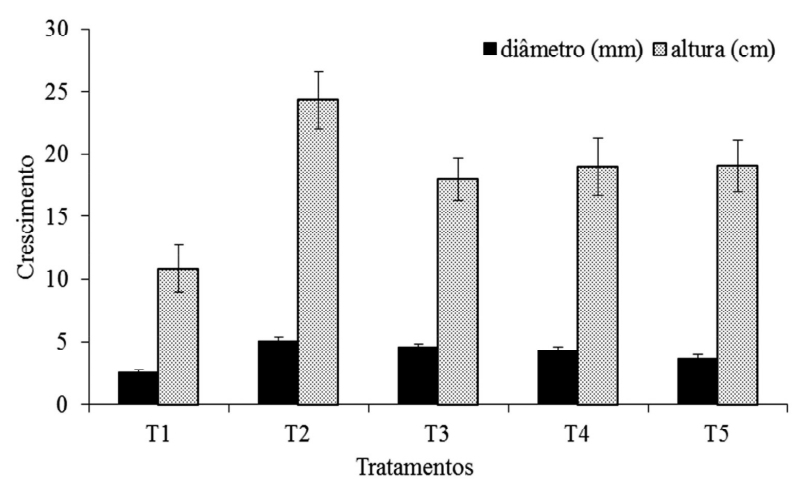

Figura 1 - Crescimento médio em diâmetro $(\mathrm{mm})$ e altura (cm) das mudas incorporadas nos diferentes tratamentos de solo, na Reserva da COPASA (Juramento, MG), após dois anos de plantio (dezembro/2001 a janeiro/2004). T1 = áreas semeadas com capim; $\mathrm{T} 2=$ covas tratadas com condicionador de solo; T3 = áreas semeadas com capim consorciado com leguminosa; T4 = áreas semeadas com leguminosa; e T5 = controle.

Figure 1 -Average diameter $(\mathrm{mm})$ and height $(\mathrm{cm})$ plant growth of seedlings at different soil treatments, in the COPASA Reserve (Juramento, $M G$ ) after two years of planting (December/2001 to January/2004). $T 1=$ areas sown with grass; $T 2=$ pits treated with soil conditioner; T3 $=$ areas sown with grass and legumes together; $T 4=$ areas sown with legumes; and $T 5=$ control.

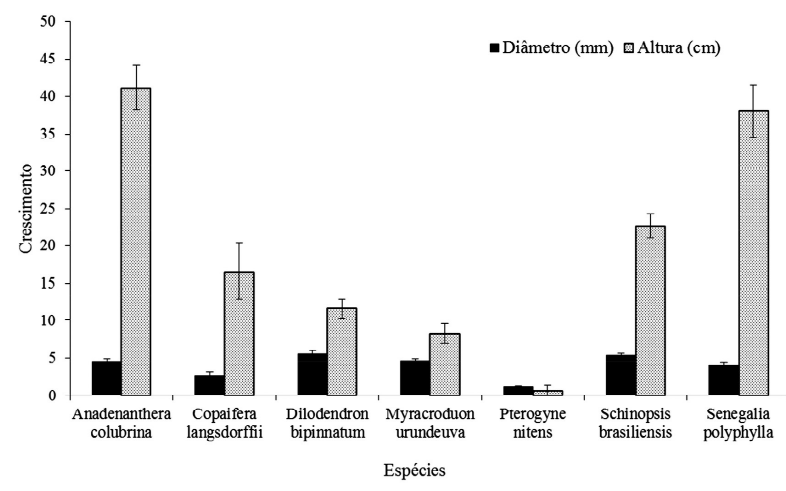

Figura 2 -Crescimento médio em diâmetro $(\mathrm{mm})$ e altura $(\mathrm{cm})$ das sete espécies incorporadas na Reserva da COPASA (Juramento, MG), após dois anos de plantio (dezembro/2001 a janeiro/2004).

Figure 2-Average diameter $(\mathrm{mm})$ and height $(\mathrm{cm})$ plant growth of seven species used in the COPASA Reserve (Juramento, $M G$ ) after two years of planting (December/2001 to January/ 2004).

Revista Árvore, Viçosa-MG, v.39, n.5, p.801-810, 2015 fisiologia da espécie, assim como das interações bióticas. Nesse experimento, embora tenham ocorrido variações interespecíficas, a mortalidade foi consideravelmente baixa (CORRÊA; CARDOSO, 1998) e concentrada nos primeiros meses após o plantio, corroborando com autores que afirmaram que a mortalidade costuma ser maior nos primeiros estágios de estabelecimento e desenvolvimento das plantas (MCDONALD et al., 2003; MARTINS, 2009). McDonald et al. (2003), em estudo realizado com espécies nativas implantadas em área de recomposição na Jamaica, encontraram mortalidade maior que a deste experimento (52 a $61 \%$ após 12 meses de avaliação), afirmando ser essa uma margem satisfatória de mortalidade para um experimento de recomposição da vegetação. Da mesma forma, Rezende (2004), em trabalho de recuperação de matas de galeria em 88 propriedades rurais no Distrito Federal e no seu entorno, obteve dados de mortalidade maiores que os deste estudo, ficando a sobrevivência geral em $16,26 \%$.

As espécies tropicais geralmente agrupam-se em relação às suas exigências ambientais e aos locais onde se adaptam, formando grupos ecológicos ou funcionais. Existem várias classificações para esses grupos, que geralmente se baseiam na tolerância ou exigência de sombra e de luz das espécies (BUDOWSKI, 1965; HARTSHORN, 1980; BROKAN, 1985; SWAINE; WHITMORE, 1988). Entretanto, critérios ecofisiológicos (SCHUPP et al., 1989), demográficos (MARTINEZRAMOS et al., 1989) e de mecanismos de dispersão de sementes (HARPER, 1977) também podem ser utilizados. Desse modo, as espécies arbóreas consideradas pioneiras, que são heliófitas (de grandes clareiras), de crescimento rápido e de pouca exigência de solo, demonstram maior aptidão a locais degradados. Assim, S. brasiliensis e A. colubrina, espécies com menor porcentagem de mortalidade neste estudo, apresentaram-se na literatura como pioneiras, secundárias iniciais ou clímax exigentes de luz (LORENZI, 1992; CARVALHO, 2003; 2008). Consequentemente, a maior sobrevivência dessas espécies no experimento pode estar relacionada ao seu carácter heliófito, o que as torna mais aptas e resistentes às condições adversas do ambiente degradado.

Espécies clímax são, no entanto, mais exigentes quanto às condições ambientais e possuem crescimento lento(BUDOWSKI, 1965; HARTSHORN, 1980; BROKAN, 1985; SWAINE; WHITMORE, 1988). Assim, a mortalidade 
mais expressiva em $C$. langsdorffii e $P$. nitens pode estar relacionada às características ecológicas apresentadas por essas espécies, já que são consideradas não pioneiras ou clímax (LORENZI, 1992; CARVALHO, 2003). Segundo Swaine e Whitmore (1988), espécies clímax tolerantes à sombra, em condições naturais, permanecem sob o dossel florestal, em locais sombreados, onde a luminosidade é bastante reduzida, a umidade do ar é mais alta e a temperatura, amena. Assim, essas espécies sofrem maior impacto quando transferidas para áreas abertas a pleno sol, principalmente por não suportarem aumento da radiação solar, da temperatura e da diminuição da umidade (VIANI; RODRIGUES, 2007).

A porcentagem de indivíduos mortos das diferentes espécies dentro dos tratamentos não apresentou padrão claro. Segundo Corrêa e Cardoso (1998), as principais causas de mortalidade de mudas plantadas em áreas degradadas são o estresse hídrico, a competição com ervas invasoras e o ataque de formigas. De forma geral, o consórcio entre capim e a leguminosa (T3) apresentou menor porcentagem de mortalidade das mudas e pode ter ocasionado um ambiente menos severo, em termos de umidade/temperatura, disponibilização de nutrientes (fixação de nitrogênio) e proteção (predadores e danos físicos), para o estabelecimento dos indivíduos implantados, apresentando maior taxa de sobrevivência das mudas. Beltrame e Rodrigues (2008), em estudo na região de Mata Atlântica, cujo objetivo foi avaliar alternativas para acelerar a restauração em florestas tropicais, testaram diferentes densidades de leguminosa (andu) consorciada com espécies nativas, e os tratamentos com essa espécie apresentaram mortalidade menor quando comparados com o controle. Entretanto, neste estudo, C. langsdorffi, D. bipinatum e $S$. polyphylla mostraram taxas de sobrevivência maiores em outros tratamentos.

O fato de o crescimento em altura e diâmetro das espécies ser maior no tratamento com condicionador de solo (T2) pode estar relacionado às propriedades desse polímero, que é um hidroabsorvente biodegradável que possui a capacidade de retenção de água e nutrientes no solo, melhorando a condição hídrica e nutricional para as mudas (VENTUROLI; VENTUROLI, 2011). De fato, a região onde está localizada a área de estudo (semiárido mineiro) possui estresse climático bastante acentuado, chegando a sete meses de extremo déficit hídrico (NUNES et al., 2005; 2012). Desse modo, o uso do condicionador de solo pode garantir a manutenção hídrica por um período maior, propiciando, com isso, desenvolvimento maior nas mudas submetidas a esse tratamento (VENTUROLI; VENTUROLI, 2011). Apesar disso, foi observada maior mortalidade de mudas nesse tratamento. Assim, o condicionador de solo pode incrementar o crescimento das espécies, tanto em diâmetro quanto em altura, mas não garante o sucesso no estabelecimento, período em que as características ecológicas aliadas à severidade das condições ambientais em áreas degradadas parecem ser fator primordial (VIANI; RODRIGUES, 2007). Como a mortalidade foi maior nos primeiros quatro meses após o plantio (em dezembro), período que coincide com a estação chuvosa e transição para a seca na região, o uso do condicionador de solo pode ter proporcionado excesso de umidade às mudas. Desse modo, como as espécies estudadas são típicas do Cerrado e da Floresta Estacional Decidual (LORENZI, 1992; POTT; POTT, 1994; CARVALHO, 2003; 2008), elas podem não apresentar adaptação a solos muito encharcados, dificultando seu estabelecimento inicial e causando, portanto, maior mortalidade. Além disso, o uso do condicionador de solo provavelmente não determina diferenças para o estabelecimento inicial, mas pode ser bastante promissor para auxiliar o crescimento, proporcionando umidade no solo por um período maior durante a estação seca.

Apesar de o tratamento com capim consorciado com a leguminosa ter garantido condições melhores ao estabelecimento das espécies, o resultado da utilização do capim isolado (sem a leguminosa) não foi satisfatório para o desenvolvimento das mudas. Esse fato pode ter sido ocasionado pela maior competição da Brachiaria sp. com as mudas implantadas, como conhecido por alguns autores, que citaram que a alta competitividade desses capins africanos pode prejudicar o desenvolvimento das mudas (MARTINS et al., 2004; ROSSI et al., 2010).

O crescimento das espécies arbóreas em áreas degradadas, principalmente nas áreas que perderam as camadas superficiais de solo, é inferior ao desenvolvimento em solos não degradados, mesmo quando esse solo recebe tratamentos físico e químico (CORRÊA; CARDOSO, 1998). Mesmo diante da degradação do solo, em que as camadas superficiais da Área de Empréstimo foram totalmente retiradas (cerca de $10 \mathrm{~m}$ de altura de solo), houve crescimento em altura e diâmetro dos indivíduos, embora relativamente baixo quando comparado a outros experimentos. Paiva e

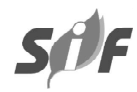

Revista Árvore, Viçosa-MG, v.39, n.5, p.801-810, 2015 
Poggiani (2000), em experimento que avalia o crescimento de cinco espécies nativas implantadas no sub-bosque de um fragmento florestal na Usina Hidroelétrica de São Joaquim, Município de Guará, SP, encontraram valores semelhantes para o crescimento em altura $(19,88$ $\mathrm{cm})$ e maiores para o crescimento em diâmetro $(2,0 \mathrm{~mm})$, quando comparados com o deste estudo. Entretanto, é importante ressaltar que, no experimento supracitado, as espécies receberam grande quantidade de adubação química e esterco bovino.

A escolha das espécies adaptadas às condições locais é um fator que contribui diretamente para o sucesso do projeto de restauração ambiental (CORRÊA; CARDOSO, 1998; TEIXEIRA et al., 2002). De fato, variações interespecíficas foram observadas possivelmente associadas à capacidade adaptativa $\mathrm{e}$ às histórias de vida das espécies em questão. As maiores médias de crescimento em altura foram apresentadas por A. colubrina e $S$. polyphylla, descritas como espécies pioneiras, rústicas e de rápido crescimento (LORENZI, 1992; CARVALHO, 2003; 2008), mostrando maior investimento no crescimento da parte aérea. O crescimento em diâmetro foi maior em $D$. bipinnatum e $S$. brasiliensis, o que permite inferir que essas espécies provavelmente investem no desenvolvimento do sistema radicular. Pterogyne nitens apresentou as menores taxas de crescimento, tanto em altura quanto em diâmetro. Segundo Carvalho (2003), P. nitens, em plantios experimentais, apresenta melhor crescimento em solos com fertilidade química elevada, bem drenados e de textura argilosa. Desse modo, as condições adversas da área de plantio, onde houve retirada de boa parte do solo, podem ter ocasionado baixa performance dessa espécie.

Corrêa e Cardoso (1998) ressaltaram que a identificação de espécies nativas, com bom desenvolvimento em áreas degradadas, é importante passo na implantação de um projeto de restauração. Assim, a meta da restauração ambiental é reconstituir um novo sistema, o mais semelhante possível do original, de modo a criar condições de autossustentabilidade, pela regeneração natural, manutenção das populações regeneradas artificialmente e garantia de diversidade genética, que possibilite a continuidade evolutiva das espécies (KAGEYAMA; GANDARA, 2003). Esse é o fundamento do desenvolvimento de projetos que visem à preservação da biodiversidade, manutenção da qualidade de vida das comunidades e desenvolvimento sustentável.

\section{CONCLUSÃO}

Apesar das variações específicas, a sobrevivência geral das mudas, após dois anos de plantio, foi alta, haja vista a adversidade do sítio de restauração e dos fatores externos, como o pisoteio de animais domesticados. Entre as espécies, A. colubrina e $S$. brasiliensis podem ser recomendadas para o plantio em ambientes degradados, em virtude da alta sobrevivência e crescimento apresentados. No entanto, $P$. nitens mostra fragilidade a ambientes comprometidos pela degradação. O tratamento com o condicionador de solo propicia maior crescimento das mudas, mas interfere na sobrevivência durante os primeiros meses de plantio. Além disso, o uso de espécies exóticas de capim mostra-se desvantajoso, por ocasionar maior mortalidade de mudas.

\section{AGRADECIMENTOS}

À COPASA S.A., pelo financiamento do projeto e pelo apoio logístico; ao CNPq, à CAPES e à FAPEMIG, pela concessão de bolsas; à UNIMONTES, pelo apoio logístico; e aos estagiários do Laboratório de Ecologia Vegetal da UNIMONTES, pelo auxílio em campo.

\section{REFERÊNCIAS}

ALVES, M.C.; NASCIMENTO, V.; SOUZA, Z.M. Recuperação em área de empréstimo usada para construção de usina hidrelétrica. Revista

Brasileira de Engenharia Agrícola e Ambiental, v.16, n.8, p.887-893, 2012.

ALVES, M.C.; SOUZA, Z.M. Recuperação de área degradada por construção de hidroelétrica com adubação verde e corretivo. Revista

Brasileira de Ciências do Solo, v.32, n.6, p.2505-2516, 2008.

ANDEL, J.V.; ARONSON, J. Getting started. In: ANDEL, J.V.; ARONSON, J. (Ed.). Restoration ecology: the new frontier. Chichester: WileyBlackwell, 2012.p.3-8.

BARON, J.S.; POFF, N.L.; ANGERMEIER, P.L.; DAHM, C.N.; GLEICK, P.H.; HAIRSTON, N.G.; JACKSON, R.B.; JOHNSTON, C.A.; RICHTER, B.D.; STEINMAN, A.D. Meeting ecological and societal needs for freshwater. Ecological Applications, v. 12, n.5, p.1247-1260, 2002. 
BELTRAME, T.P.; RODRIGUES, E. Comparação de diferentes densidades de feijão guandu (Cajanus cajan (L.). Millsp.) na restauração florestal de uma área de reserva legal no Pontal do Paranapanema, SP. Scientia Florestalis, v. 36 , n. 80, p.317-327, 2008.

BROKAN, N.V.L. Gap-phase regeneration in a tropical forest. Ecology, v.66, n.3, p.682-687, 1985.

BUDOWSKI, G. Distribution of tropical American rain forest species in light of successional processes. Turrialba, v. 15, n.1, p.40-42, 1965.

CARVALHO, P.E.R. Espécies arbóreas brasileiras. v. 1. Brasília, DF: Embrapa Informação Tecnológica, 2003. 1039p.

CARVALHO, P.E.R. Espécies arbóreas brasileiras. v. 3. Brasília: Embrapa Informação Tecnológica, 2008. 593 p.

CHADA, S.S.; CAMPELLO, E.F.C.; FARIA, S.M. Sucessão vegetal em uma encosta reflorestada com leguminosas arbóreas em Angra dos Reis, RJ. Revista Árvore, v.28, n.6, p.801-809, 2004.

CLEWELL, A.F.; ARONSON, J. Ecological restoration: principles, values and structure of an emerging profession. Washington: Island Press, 2013. 303p.

CORRÊA, R.S.; CARDOSO, E.S. Espécies testadas na revegetação de áreas degradadas. In: CORRÊA, R.S.; MELO-FILHO, B. (Org.) Ecologia e recuperação de áreas degradadas no cerrado. Brasília, DF: Paralelo, 1998. p.101-116.

DAVIES, P.M. Climate change implications for river restoration in global biodiversity hotspots.

Restoration Ecology, v. 18, n.3, p.261-268, 2010.

EMPRESA BRASILEIRA DE PESQUISA AGROPECUÁRIA - EMBRAPA. Sistema brasileiro de classificação de solos. Brasília, DF: Embrapa, 2013.353p.

GARCÍA-ORTH, X.; MARTÍNEZ-RAMOS, M. Isolated trees and grass removal improve performance of transplanted Trema micrantha (L.) Blume (Ulmaceae) saplings in tropical pastures. Restoration Ecology, v.19, n.1, p.24-34, 2011
GARWOOD, N.C. Functional morphology of tropical tree seedlings. In: SWAINE, M.D. (Ed.). The ecology of tropical forest tree seedlings. Paris: UNESCO, 1996. p.59-129.

HARPER, J.L. Population biology of plants. London: Academic Press, 1977. 892p.

HARTSHORN, G.S. Neotropical forest dynamics. Biotropica, v.12, n.1, p.23-30, 1980.

KAGEYAMA, P.; GANDARA, F.B. Restauração e conservação de ecossistemas tropicais. In: CULLEN JR., L.; RUDRAN, R.; VALADARESPADUA, C. (Org.) Métodos de estudos em biologia da conservação da vida silvestre. Curitiba: UFPR/Fundação O Boticário de Proteção à Natureza, 2003. p.383-394.

LORENZI, H. Árvores brasileiras: manual de identificação e cultivo de plantas arbóreas nativas do Brasil. São Paulo: Plantarum, 1992. v.1.368p.

MARTINEZ-RAMOS, M.; ALVAREZ-BUYLLA, E.; MARTINS, S.E. Tree demography and gap dynamics in a tropical rain forest. Ecology, v.70, n.3, p.555-558, 1989.

MARTINS, C.R.; LEITE, L.L.; HARIDASSAN, M Capim-gordura (Melinis minutiflora P. Beauv.), uma gramínea exótica que compromete a recuperação de áreas degradadas em unidades de conservação. Revista Árvore, v.28, n.5, p.739-747, 2004.

MARTINS, S.V. Recuperação de áreas degradadas: ações em áreas de preservação permanente, voçorocas, taludes rodoviários e de mineração. Viçosa, Mg: Aprenda Fácil, 2009. 270p.

MCDONALD, M.A.; HOFNY-COLLINS, A.; HEALEY, J.R.; GOODLAND, T.C.R. Evaluation of trees indigenous to the montane forests of the Blue Mountains, Jamaica for reforestation and agroforestry. Forest Ecology and Management, v. 175, p.175-379, 2003.

MELO, G.A.; D'ANGELO-NETO, S.; ROCHA, I.D.F.; MIRANDA, A.A.; FONSECA, C.C.; NERI, A.V. Caracterização fitossociológica das matas ciliares da represa da COPASA no Município de Juramento (MG). Relatório Técnico. Montes Claros: Universidade Estadual de Montes Claros, 2000.

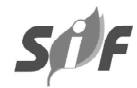

Revista Árvore, Viçosa-MG, v.39, n.5, p.801-810, 2015 
MÜLLER, A.C. Hidrelétricas, meio ambiente e desenvolvimento. São Paulo: Makon Books, 1995. 412p.

NUNES, Y.R.F.; FAGUNDES, M.; SANTOS, R.M.; DOMINGUES, E.B.S.; ALMEIDA, H.S.; GONZAGA, A.P.D. Atividades fenológicas de Guazuma ulmifolia Lam. (Malvaceae) em uma Floresta Estacional Decidual no norte de Minas Gerais. Lundiana, v.6, n.2, p.99-105, 2005.

NUNES, Y.R.F.; LUZ, G.R.; BRAGA, L.L. Phenology of tree species populations in tropical dry forests of Southeastern Brazil. In: ZANG, X. (Ed.). Phenology and climate change. Rijeka: Intech, 2012. p.125-142.

PAIVA, A.V.; POGGIANI, F. Crescimento de mudas de espécies arbóreas nativas plantadas no subbosque de um fragmento florestal. Scientia Forestalis, v.57, p.141-151, 2000.

PALMER, M.; ALLAN, D.; MEYER, J.; BERNHARDT, E.S. River restoration in the twenty-first century: data and experiential knowledge to inform future efforts. Restoration Ecology, v.15, n.3, p.472-481, 2007.

POTT, A.; POTT, V.J. Plantas do Pantanal. Brasília: Embrapa, 1994. 320 p.

R DEVELOPMENT CORE TEAM. R: A language and environment for statistical computing. Vienna: R Foundation for Statistical Computing. Disponível em: http://www.R-project.org. Acessado em 10 de jun. de 2014.

REZENDE, R.P. Recuperação de matas de galeria em propriedades rurais do Distrito Federal e entorno. 2004. $145 \mathrm{f}$. Dissertação (Mestrado em Ciências Florestais) Universidade de Brasília, Brasília, 2004.

RODRIGUES, G.B.; MALTONI, K.L.; CASSIOLATO, A.M.R. Dinâmica da regeneração do subsolo de áreas degradadas dentro do bioma
Cerrado. Revista Brasileira de Engenharia Agrícola e Ambiental, v.11, n.1,p.73-80, 2007.

RONI, P.; BEECHIE, T. Stream and watershed restoration: a guide to restoring riverine processes and habitats. Oxford: WileyBlackwell, 2013.

ROSSI, R.D.; FIGUEIRA, J.E.C.; MARTINS, C.R. Capim-gordura, invasão biológica, conservação do cerrado e regime de fogo. MG- Biota, v.3, p.427,2010

SANSEVERO, J.B.B.; PRIETO, P.V.; MORAES, F.D.; RODRIGUES, P.J.F.P. Natural regeneration in plantations of native trees in lowland Brazilian Atlantic Forest: community structure, diversity, and dispersal syndromes. Restoration Ecology, v.19, p.379-389, 2011

SCHUPP, E.W.; HOWE, H.F.; AUGSPURGER, C.K. Arrival and survival in tropical treefall gaps. Ecology, v.70, p.562-564, 1989.

SWAINE, M.D.; WHITMORE, T.C. On the definition of ecologial species groups in tropical rain forest. Vegetatio, v.75, n.1, p.81-86, 1988.

TEIXEIRA, M.C.B.; NUNES, Y.R.F.; PEDRALLI, G. SOUZA, G.P.; SANTOS, J.C.; GUIMARÃESFILHO, P.E.; MAIA, K.M.P. Recomposição da cobertura vegetal em áreas degradadas na Área de Proteção Especial da Mutuca (APE-Mutuca), Belo Horizonte-MG. Revista Estudos de Biologia, v.24, n.48, p.51-59, 2002.

VENTUROLI, F.; VENTUROLI, S. Recuperação florestal em uma área degradada pela exploração de areia no distrito federal. Ateliê Geográfico Goiânia-GO, v.5, n.13, p.183-195, 2011.

VIANI, R.A.G.; RODRIGUES, R.R. Sobrevivência em viveiro de mudas de espécies nativas retiradas da regeneração natural de remanescente florestal. Pesquisa Agropecuária Brasileira, v.42, n.8, p.1067-1075, 2007. 